\title{
Ein keltisches Versmass im Provenzalischen und Französischen.
}

In Lemcke's Jahrbuch I 2, 5 habe ich die Wahrscheinlichkeit darzuthun versucht, dass wir in der bekannten bei dem ältesten Troubadour und in altfranzösischer volksthümlicher Poesie vorkommenden Strophenform eine keltische Volksweise haben. Das Gleiche gilt, wie ich im Folgenden zeigen will, von der zweiten Lieblingsform des Grafen von Poitou, welche von seinen Liedern nicht weniger als drei haben. Ich meine den elfsilbigen Vers, mit einer männlichen oder weiblichen Cäsur nach der siebenten (achten) Silbe. ${ }^{1}$

Betrachten wir zuerst die Behandlung des Verses in diesen drei Liedern (Grundriss I 83, 3. 4. 5). In jedem werden drei Verse zu einer einreimigen Strophe verbunden, von denen jedoch der dritte ein Vers von fünfzehn Silben ist, mit einer weiblichen Cäsur nach der achten Silbe. Der Reim der ersten Strophe geht durch alle folgenden hindurch. Man könnte die Verse trochäische nennen, wenn dies nicht den noch jetzt oft gehörten und gelesenen Irrthum zu nähren geeignet wäre, dass die romanischen Verse nach Füssen und nicht vielmehr silbenzählend-accentuirend gebaut seien. Ich bezeichne daher im folgenden Schema die indifferenten Versstellen, d. h. die, bei denen der Accent beliebig fallen kann, durch Punkte, die nothwendig betonten durch _- und die nothwendig nicht betonten durch $\smile$. Demgemäss ist das Schema des kürzeren Verses entweder $\quad \cdot \quad \cdot \quad \cdot \cdot+1 \cdot . \cdot 1$

Beispiel :

oder

E tengatz lo per vilan | qui no l'enten

$$
\text { Dos cavals ai a ma selha|ben e gen. }
$$

Mitunter kommt es aber vor, dass auch bei männlicher Cäsur die zweite Hälfte nur drei Silben hat. So 183, 3:

$$
\begin{aligned}
& \text { Companho, farai un vérs | covinen } \\
& \text { o dins son cor voluntiérs | non l'apren. }
\end{aligned}
$$

Bei weiblicher Cäsur hat die zweite Hälfte immer drei Silben. Eine scheinbare Ausnahme ist 183,3 , Str. 5:

mas tan fera estranheza | a longamen;

' Vgl. Diez, Altromanische Sprachdenkmale S. $123 \mathrm{f}$. 
allein hier findet Elision über die Cäsur hinüber statt. Dasselbe auch 183,4 , Str. 5 .

Aehnlichen Bau zeigt auch der längere fünfzehnsilbige Vers. Das gewöhnliche Schema desselben ist

$$
\text { et es tan fers e salvátges| que del baillar si defén. }
$$

Auch hier kommt daneben die männliche Cäsur, doch selten, vor, ohne dass deswegen die zweite Hälfte eine Silbe mehr als bei männlicher hat, wie dies die Regel in dem kürzeren Verse ist. So in 183,3 :

$$
\begin{aligned}
& \text { et er totz mesclatz d'amór | e de joi e de joven. } \\
& \text { greu partir si fai d'amór | qui la trob' a son talen. } \\
& \text { mas nols posc amdos tenér | que l'us l'autre non consen. } \\
& \text { que meills for' encavalgátz | de nuill autr' ome viven.' } \\
& \text { aquest non er ja camjátz | ni per aur ni per argen. } \\
& \text { que s'il lo tenia un d́n | qu'eu lo tengues mais de cen. } \\
& \text { c'ambedui me son jurát |e plevit per sagramen. }
\end{aligned}
$$

Wahrscheinlich auch 183,4 , Str. 6:

si non pot aver cavál|. . cumpra palafrei,

wiewohl hier die Lücke auch nach aver angenommen und drei Silben ergänzt werden könnten.

Fehlerhaft gebaut ist MG. I72, 5. 3 per un altre c'om hi tailla | $n^{\prime} i$ naison dos o treis, wo der zweiten Hälfte eine Silbe fehlt; wahrscheinlich ist en $i \mathrm{zu}$ schreiben.

In dieser einfachsten Gestalt kommt die Strophe sonst nirgend vor. Das auch dem Alter nach nächste Beispiel liefert ein Lied von Marcabrun (Gr. 293, 24), in welchem jedoch alle drei Zeilen der Strophe durch Binnenreim gebrochen sind. Die Art, wie das Lied nach A von Grützmacher im Archiv 33, 334 abgedruckt ist, lässt freilich die Aehnlichkeit nicht erkennen. Sie ist zu schreiben:

$$
\begin{aligned}
& \text { En abriu s'esclairoil riu contral pascor, } \\
& e \text { per lo broill naissoil foill sobre la flor } \\
& \text { belamen ab solatz gen ab conort de fin' amor. }
\end{aligned}
$$

Hier haben wir vollständig die Strophe wie beim Grafen von Poitou, drei auf einen männlichen, durch alle Strophen beibehaltenen Reim (auf or) ausgehende Zeilen, deren dritte länger ist. Den einzigen Unterschied macht der Binnenreim. Dass solcher anzunehmen, beweist 1) der Wechsel des Reimgeschlechtes, 2) der Wechsel der Stellung und 3) die mehrfach vorkommende Elision über den Reim hinüber. ${ }^{2}$ Wechsel des Reimgeschlechtes findet sich bei dem kürzeren wie bei dem längeren Verse:

und mit Elision:

blanc lo teigna pois lo deigna ses brunor,

Deus maliga amor piga e sa valor. si la mia non crezia enganador.

'Die zweite Vershälfte lautet in $\mathrm{E}$ : de nuill home uiuen, in $\mathrm{C}$ nur: dome uiven.

2 Vgl. Germania 12, 148-155. 
In der längeren Versart:

mit Elision:

seus seria sim volia ses bauzia e ses error,

$$
\text { c'amors vaire al meu vejaire a l'uzatge al trahidor. }
$$

Wechsel der Stellung. In der kürzeren Versart ausser der ersten Strophe, wo man freilich durch $e$ pel broill naisso li foill die gewöhnliche Stellung leicht erzielen könnte, noch in folgendem Verse: ans de totz malvatz pren patz cals l'a groissor.

In dem längeren Verse:

vers es per ben fait cap frait en mains locs es per honor.

Der längere Vers hat, auch bei männlicher Cäsur, in der zweiten Hälfte nur sieben Silben, also genau wie beim Grafen von Poitou; der kürzere bei weibiicher Cäsur in der zweiten Hälfte drei Silben (scheinbare Ausnahmen macht auch hier nur Elision), bei männlicher vier Silben, nicht auch zuweilen drei, wie beim Grafen.

Diez führt (S. I 23 f.) ausser den Liedern des Grafen noch ein zweites provenzalisches Beispiel an, die Tenzone zwischen Bertran und Augier (Gr. 205, I). Hier bilden drei elfsilbige, einreimige Zeilen den Anfang der Strophe, am Schlusse folgen vier kürzere Verse. Ich gebe als Probe eine der weniger entstellten Strophen:

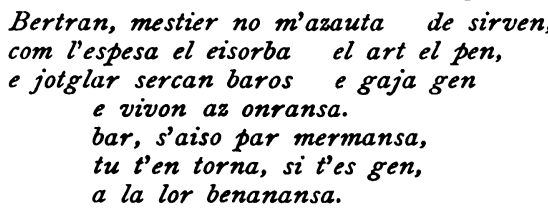

Die Behandlung des elfsilbigen Verses ist dieselbe wie die früher bemerkte. Die Cäsur kann männlich oder weiblich sein, Elision über die Cäsur ist gestattet; bei männlicher Cäsur muss die zweite Hälfte immer vier Silben haben. Die Reime wechseln von zwei zu zwei Strophen. Bemerkenswerth ist der erste Vers der Tenzone

\section{Bertran, vos c'anar sollatz ab lairos,}

wegen der auffallenden Betonung des Verbums. Ganz allein steht dieselbe indessen nicht; auch Guiraut Riquier braucht poiriatz : fariutz: veziatz: auzirlatz als weibliche Reime (62, 93. 95. 103. 105); ebenso in der ersten Person estariam : caziam ( 7 I, 3 I I f.).

Wie in der eben angeführten Tenzone, so werden auch in einer Tenzone zwischen Auzer Figueira und Aimeric von Peguillan (Gr. 42, 3) drei Zeilen, auf einen männlichen Reim ausgehend, mit vier darauf folgenden kürzeren Zeilen zu einer Strophe verbunden:

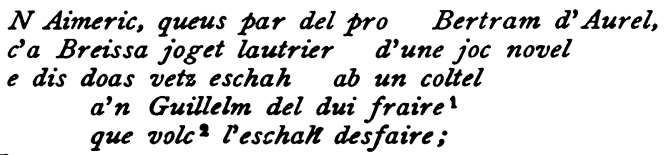

${ }^{1}$ In den Worten del dui fraire scheint ein Name zu stecken.

2 Hs. uol. 
mas Bertrams levet del joc

can Guillelms cuidet traire.'

Die Cäsur kann auch weiblich ausgehen (Str. 2, 2) laises joc :obrel maiestre | d'en Sorilel.

Drei Zeilen auf einen durch alle Strophen gehenden männlichen Reim, aber mit Inreimen nach der dritten und siebenten Silbe lässt Raimbaut von Aurenga (Gr. 389,3 ) die Strophe beginnen und dann ebenfalls kürzere Verse folgen:

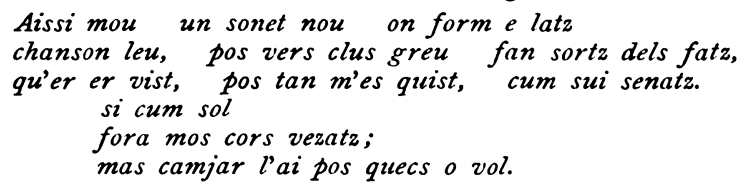

Mit Inreimen nach der siebenten Silbe, also die Cäsuren unter cinander gereimt, verbindet drei einreimige elfsilbige Verse Ramon de Tors (Gr. 4IO, 5) und schliesst daran vier auf einen männlichen Reim ausgehende Verse von sieben Silben:

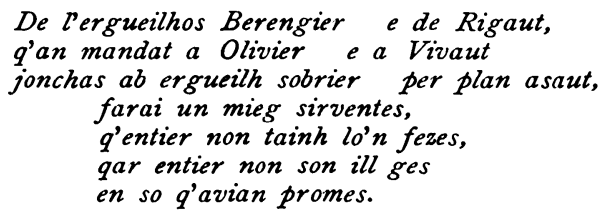

Die Reime wechseln mit jeder Strophe, die Cäsuren sind nur männlich.

Auch ein Tanzlied von Uc de S. Circ (Gr. 457, 4I) ist in der elfsilbigen Versart abgefasst. Die Strophe beginnt ebenfalls mit drei solcher Verse, die in den Cäsuren gereimt sind, kürzere Verse bilden den Schluss:

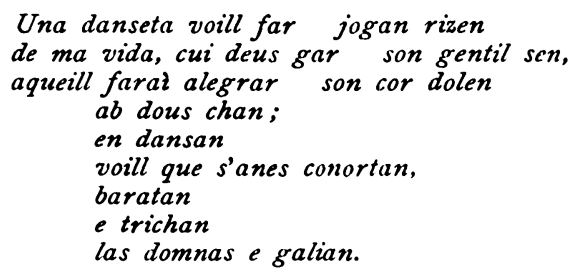

Vicr derartige Zeilen, auf einen männlichen Reim ausgehend, worauf kürzere Verse folgen, bilden die Grundlage der Strophe in einer Tenzone zwischen Guillelm Raimon und Aimeric von Peguillan (Gr. 229, 2):

1 Die Strophenform dieser Tenzone ist dieselbe wie die der oben angeführten Tenzone zwischen Augier und Bertran. Diese bezeichnet Diez (Sprachdenkm. I23) als von 'zweien der ältesten Sänger' herrührend; er erblickt mithin in Augier den alten Dichter des 12. Jahrh. Ich bin ihm darin gefolgt (Gr. 205, I), wie ich jetzt glaube, mit Unrecht. Augier ist vielmehr identisch mit Auzer Figueira, Auzer ist norditalienische mundartliche Form für Augier (vgl. Zorgi für Gorgi). Unter Bertran kann Bertran d'Alamano, aber auch Bertram d'Aurel verstanden werden. 


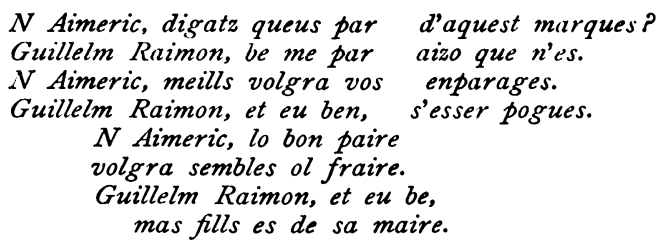

Die Strophenform ist genau dieselbe wie in den beiden vorher erwähnten Tenzonen (S. I 97 f. und Anm.), nur dass hier eine Langzeile mehr. Der Grund diescr Hinzufügung liegt in dem Wechsel der redenden Personen innerhalb derselben Strophe; da jeder dreimal das Wort nimmt, muss die Zahl der Verse eine gerade sein.

Bisher haben wir nur männliche Endreime gefunden. Aber auch mit weiblichem Ausgang kommt die elfsilbige Versart vor. So in einem Sirventes von Guillem de Berguedan (Gr. 2 IO, 2 I), dessen erste Strophe lautet:

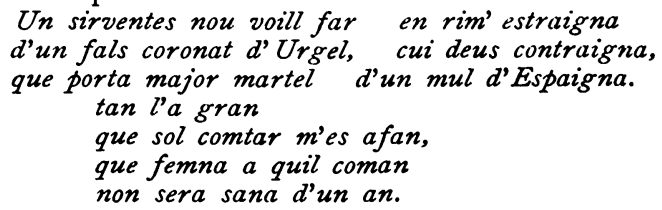

Die Reime wechseln in jeder Strophe: die Cäsuren sind durchaus männlich. Der Reim in der Cäsur der zweiten und dritten Zeile kehrt auch in der fünften Strophe wieder (lejal : porpal); eine Durchführung ist jedenfalls nicht beabsichtigt. Bemerkenswerth ist, dass der Dichter sagt, er wolle ein neues Sirventes dichten en rim' estraigna. Letzterer Ausdruck bezieht sich nicht auf den Reim, sondern auf die angewandte Versart. Denn die Reime sind nicht fremdartig, I aigna, 2 orta, 3 uga, 4 eigna, 5 ona, die männlichen I an, 2 or, 3 os, 4 orn, 5 en; von diesen könnten etwa nur orta und uga als 'rimas caras' betrachtet werden. Aber fremdartig und selten ist die angewandte Versart, welche den Grundstock der Strophe bildet, und darauf geht ausser estraigna vielleicht auch der Ausdruck nou im Sinne von latein. novus.

Gleichfalls mi. klingenden Reimen, aber in gekreuzter Stellung, lässt Peire Cardenal (Gr. 335, 44) elfsilbige Verse die Strophe eines Sirventes beginnen; es sind aber nicht drei, sondern vier Langzeilen, auf welche dann vier kürzere (siebensilbige) Zeilen folgen, die Reimpaare bilden:

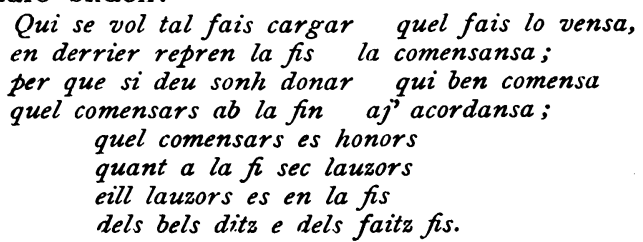

Die Handschrift C, welcher Raynouards Text (LR. I, 459) folgt, ändert hier durchgängig den elfsilbigen Vers in den zehnsilbigen 
um, ein Beweis, dass die Versart ungewohnt (estraigna) und den Schreibern anstössig war. Auch die anderen Handschriften haben vielfach Fehler, aber keine hat so systematisch geändert wie $\mathrm{C}$. In diesem Sirventes ist die Cäsur durchgängig männlich, die Reime gehen durch alle Strophen hindurch in der Stellung $\mathrm{a} b \mathrm{a}$.

In der Handschrift $\mathrm{T}$ 108b (Gr. 46I, I I 2) findet sich, mitten unter Liedern von Peire Cardenal, ein anonymes Gedicht, dessen Strophenform mit der von Peire Cardenal gebrauchten ganz übereinzustimmen scheint. Der Text ist sehr entstellt; ich führe nur die erste Strophe an:

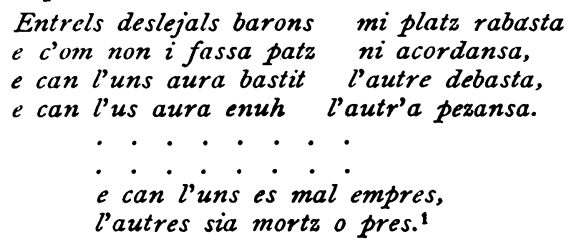

Ebenfalls mit der Strophe bei Peire Cardenal stimmt die Form einer Tenzone zwischen Bonafe und Blacatz (Gr. 98, I) überein, nur dass die vier Schlussverse nicht zwei Reimpaare, sondern einen vierfachen Reim bilden:

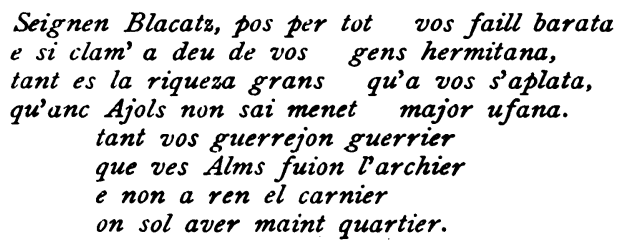

In den Handschriften fehlt in der ersten Zeile vos: man könnte auch Seigner en Blacatz (ohne vos) schreiben, allein dadurch würde die Cäsur zwischen per und tot fallen; auch empfiehlt die Analogie von 5, 1. 7, I die Beibehaltung von Seignen. Die Cäsur ist hier ebenfalls nur männlich.

Noch genauer übereinstimmend mit der Strophenform von Peire Cardenal ist dic eines Gedichtes von unanständigem Inhalt, welches von $\mathrm{C}$ dem Guillem de S. Leidier, von $\mathrm{R}$ dem Peire Duran beigelegt wird (Gr. 234, 8). Die erste Strophe lautet:

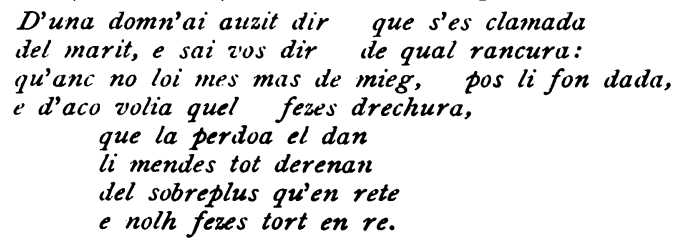

${ }^{1}$ Die Hs. hat Z. I Entels desle als. plas. 2 noi fassa pas. 3.4 sind vertauscht. 4 enuhet lautre $p$. Nach 4 (3) ist eine Zeile Raum gelassen; es fehlen zwei Reimzeilen. 
Dic Elision über die Cäsur hinüber ist gestattet (Str. 3, 3). Einmal $(4,3)$ ist die Cäsur weiblich, wobei die zweite Vershälfte eine Silbe weniger hat: qu'eu aucis que non ac autra | malautia.

Anwendung des Inreims in dem elfsilbigen Verse haben wir mehrfach gefunden; den stärksten Gebrauch davon hat Serveri (Gr. 434, 14) gemacht, wo ich die Strophe folgendermassen schreibe:

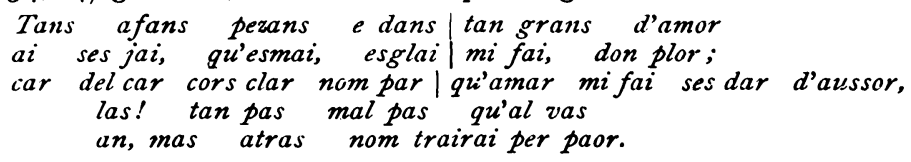

So gegliedert nimmt sie sich freilich wesentlich anders aus als bei Milá S. 378, wo nach jedem Reimwort abgesetzt ist. Wir haben zwei elfsilbige und einen fünfzehnsilbigen Vers mit einander verbunden, also genau dieselbe Form wie beim Grafen von Poitou. Der Unterschied besteht ausser dem Anhang von kürzeren Versen, dem wir schon oft begegnet sind, in der Behandlung des fünfzehnsilbigen Verses. Dieser hat hier, was mit dem Inreim zusammenhängt, nur männliche Cäsur, aber dann in der zweiten Hälfte nicht sieben, sondern acht Silben, während beim Grafen von Poitou auch bei männlicher Cäsur nur sieben (vgl. S. 196).

In allen bisherigen Beispielen fanden wir den elfsilbigen Vers, zwei-, drei- oder viermal wiederkehrend, den Anfang der Strophe bilden. Nirgend ist uns der Vers einzeln stehend begegnet. Ich wüsste dafür auch nur einen Beleg aus dem Provenzalischen anzuführen (im Französischen, wie wir sehen werden, kommt es öfter vor), und zwar durch inneren Reim gebrochen, in einem Liede Perdigons (Gr. 370, 5):

\section{quel comjatz quem fo donatz gent autrejatz.}

Die übrigen Verse der Strophe sind sieben- und dreisilbige, also solche, wie wir sie allerdings gern mit dem elfsilbigen verbunden sahen. Bedenken erweckt mir nur, dass das Ende des Verses mit dem Inreim gebunden ist, was sonst in dieser Versart nicht vorkommt.

Die Leys d'amors (I, I I6) kennen auch einen Vers von elf Silben, und die von ihnen angeführten Beispiele lassen sich zum Theil ganz gut nach dem alten Schema lesen:

$$
\begin{aligned}
& e \text { pros et arditz e fortz e vigoros. } \\
& e \text { de bon alberc guarnitz de fina malha; }
\end{aligned}
$$

auch bei weiblicher Cäsur:

$$
\begin{aligned}
& \text { l'elme ni l'escut, l'espaza, ni la lansa. } \\
& \text { no vuelha layshar e mene bon destrier. }
\end{aligned}
$$

Aber andere Verse passen nicht in das Schema, wie

las sagetas larc que de luenh fier e lanssa.

e may trompadors per mesclar las companhas etc.

Zudem trifft die Definition nicht auf den alten Vers zu. Die LA kennen nur eine Cäsur nach der fünften oder nach der sechsten Silbe; ihr Schema also ist entweder 
oder

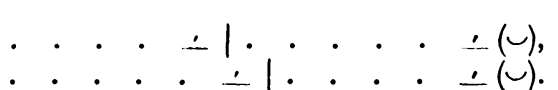

Die Verfasser kennen also offenbar die alte Versart gar nicht. Von ihren beiden Arten kommt die zweite bei den Troubadours überhaupt nicht vor; die erste Art kenne ich auch nicht als einen Vers, wohl aber in der Verbindung zweier Verse, von denen der erste bei männlichem Reime fünf, der zweite bei weiblichem Reime sieben Silben hat. Fasst man beide als einen Vers mit gereimter Cäsur, so ergibt sich genau jener elfsilbige Vers, der bei weiblichem Reime zwölf Silben zählt. Diese Verbindung hat Guillem Figueira in seinem Sirventes gegen Rom (Gr. 2 1 7, 2):

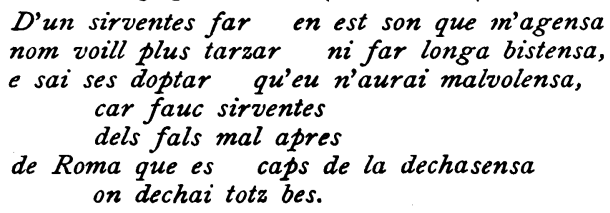

Die Aehnlichkeit der Strophenbildung mit den früher angeführten fällt in die Augen; auch hier drei längere elfsilbige Verse, auf cinen Reim ausgehend, worauf kürzere folgen; nur ist noch einmal ein längerer Vers, mit jenen dreien reimend, in den Schluss cingefügt. Dieselbe Form finden wir bei Gaucelm Faidit (Gr. 167, 2) und in einem anonymen Marienliede (Gr. 46I, 23). Mit drei Langzeilen hebt auch ein anderes Lied (Gr. 167, 55) Gaucelm Faidit an, worauf kürzere Verse folgen. In erweiterter Gestalt erscheint die Strophe bei Peire Cardenal (Gr. 335, 38), indem fünf Langzeilen sie eröffnen.

An sich wäre es gar nicht undenkbar, dass neben dem elfsilbigen Verse mit Cäsur nach der siebenten Silbe sich eine Form mit Einschnitt nach der fünften entwickelt hätte. Den gleichen Wechsel haben wir bei dem zehnsilbigen Verse, der seine Cäsuren sowohl nach der vierten als nach der sechsten Silbe hat. Die gereimte Cäsur fanden wir auch bei der Form mit Einschnitt nach der siebenten Silbe (S. 198).

In der altfranzösischen Poesie begegnet der elfsilbige Vers am häufigsten in der Pastourelle. Schon Diez hat zwei Beispiele davon angeführt (S. 1 24). In dem ersten beginnt die Strophe mit drei elfsilbigen auf einander reimenden Versen mit männlichem Ausgang, worauf ein kürzerer Vers von sechs Silben und ein dreizeiliger Refrän folgt (meine Romanzen und Pastourellen I, 64):

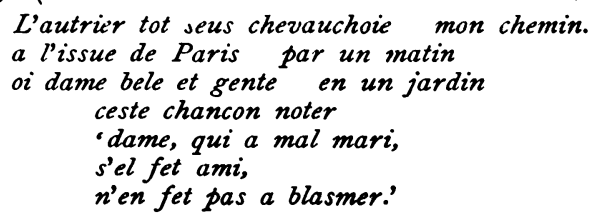


Die Cäsur kann männlich und weiblich sein, Elision über die Cäsur ist gestattet. Das zweite von Diez erwähnte Beispiel ist eine anonyme Pastourelle (II, 6I), deren erste Strophe lautet:

$$
\begin{aligned}
& \text { Hui main par un ajornant } \\
& \text { chevauchai ma mule amblant, } \\
& \text { trouvai gentil pastorele et avenant, } \\
& \text { entre ses aigniaux aloit joie menant. }
\end{aligned}
$$

Hier bildet also der elfsilbige Vers den Schluss der Strophe, die kürzeren Verse gehen voran. Die Cäsur ist auch hier männlich oder weiblich, Elision gestattet (noch V. 19. 20. 28); der Reim wechselt sein Geschlecht in den verschiedenen Strophen nach Belieben.

Ich führe ferner noch folgende Pastourellen an. II, 52:

$$
\begin{aligned}
& \text { Putepoinne chivauchoit a matinet: } \\
& \text { a l'ixue de Lowon leis un bouchet } \\
& \text { vi pastoure ou cuet muguet } \\
& \text { et faixoit un chapelet. } \\
& \text { d'amours s'escriait trois mos } \\
& \text { 'odeli odeli odeli o! } \\
& \text { dieus! amors m'ont navrei a mort.' }
\end{aligned}
$$

Nach zwei Langzeilen folgen drei siebensilbige Verse und ein zweizeiliger Refrän. Die Reime sind männlich und wechseln mit Ausnahme des Refränreims in jeder Strophe; die Cäsur geht männlich und weiblich aus.

II, IO4 stehen zwei Langzeilen als Schluss einer Strophe, die im Uebrigen aus sieben kürzeren (sieben- und achtsilbigen) Versen besteht; es ist ein Motet:

$$
\begin{aligned}
& \text { le tresor } u \text { il convient tant de tirlot, } \\
& \text { com un petitet de bien avec Marlot. }
\end{aligned}
$$

Die Cäsuren sind vielleicht ungenau gereimt.

Die Anwendung des Inreims in dieser Versart findet sich wie bei den Provenzalen. Die Cäsuren sind gereimt, theils unter einander, theils mit dem Schluss des Verses, II, 55:

$$
\begin{aligned}
& \text { E bergiers, si grant anvie j'ai de toi } \\
& \text { de ceu que si bone vie ais envers moi, } \\
& \text { c'onkes loialtei ne foi trover ne poi } \\
& \text { lai ou je l'ai deservie } \\
& \text { et tu qui de riens servie } \\
& \text { n'ais amors, joir t'an voi et vanteir t'oi } \\
& \text { an l'anoy, jus an l'anoi en bras t'amie. }
\end{aligned}
$$

Drci Langzeilen beginnen die Strophe, darauf folgen kürzerc Verse, und dann nochmals die längere Versart. Letzteres fanden wir auch in der provenzalischen Variation mit Cäsur nach der fünften Silbe (S. 202). Die Schlusszeile ist Refrän.

Gleichfalls gereimte Cäsuren hat II, I00, wo zwei Langzeilen die Strophe eröffnen und kürzere Verse nachfolgen:

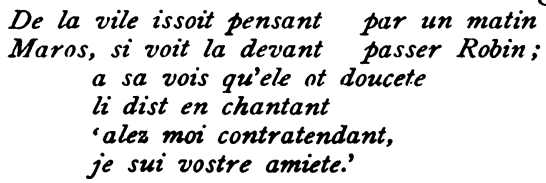


Zwei Langzeilen mit gereimten Cäsuren eröffnen, kürzere Verse von sieben Silben folgen bei Ernous li Vielle (III, 9):

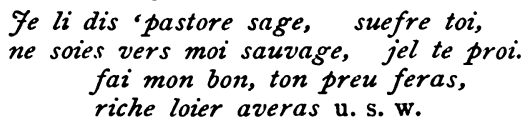

Die Inreime sind hier durchgängig weiblich, daher hat die zweite Hälfte nur drei Silben, ausgenommen wo Elision über die Cäsur stattfindet (V. 26).

Eine Langzeile mit Inreim auf der dritten und siebenten Silbe (wie im Provenzalischen mehrfach, S. 196. 198) beginnt die Strophe, kürzere Verse folgen III, I 6:

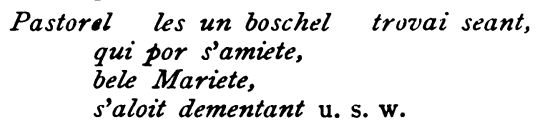

Zwei Langzeilen, jede einen der Stollen beginnend, die Cäsur und das Ende weiblich gereimt II, 35:

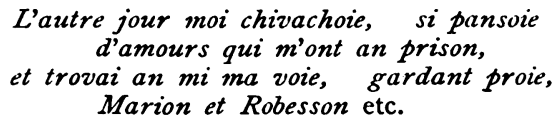

Zwei Langzeilen mit weiblichem Inreim nach der vierten und achten Silbe mitten in einer Strophe aus kürzeren Versen II, I 2 I :

$$
\begin{gathered}
\text { seuls aloie, si pensoie as noviaus sons } \\
\text { que soloie: touse gaie o ses moutons } \\
\text { trovai sanz conpegnons etc. }
\end{gathered}
$$

wo beidemal Elision über die Cäsur stattfindet.

Einzelne Langverse mitten in eine Strophe eingefügt kommen selten vor. Ein paar Beispiele gewährt II, 44, theils mit, theils ohne Inreim:

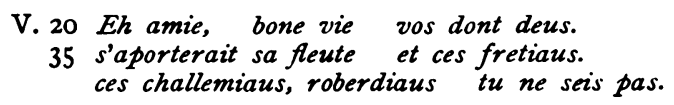

Die zweite noch häufigere Verwendung des elfsilbigen Verses ist die als Refrän von Liedern und Strophen. Diez, der mehrere Beispiele davon anführt (S. 1 24), betrachtet sie als 'Lieder- oder Strophenanfänge ganz im Volkston'. Sie können sehr wohl den Anfang des Liedes gebildet haben und doch Refräns gewesen sein; der Refrän jeder Strophe wurde dem ganzen Liede vorausgeschickt, gewissermassen als das Thema des Liedes (vgl. R. u. P. I, 23. 24. 25. 26 u. öfter). Ich gebe im Nachstehenden aus einer seit lange angelegten Sammlung altfranzösischer Refräns (vgl. R. u. P. S. Xv) hier diejenigen, die den elfsilbigen Vers zeigen.

Einer der beliebtesten Refräns ist

$$
\text { 1. c'est la fins, quoi que nus die, j'amerai, }
$$

der nicht weniger als fünfmal belegt ist: R. u. P. I, $53^{\mathrm{a}}, 8$ und meine Anmerkung. 
2. tel mari n'aves ros mie ke jou ai: il dist k'il me batera ou j'amerai.

R. u. P. I, 67, $35 \mathrm{f}$.

3. vos direz quanque voldrez, mais $j$ 'amerai.

R. u. P. I, 39, 23.

4. jamerai ceu ke m'ocist et bien le sai.

Archiv für das Studium der neueren Sprachen 43, 3 I3.

5. se cist maus ne m'assoage, je morrai.

R. u. P. III, $46,23$.

6. dieus! conment durer porai, aimi! hahai! quant a la biele que j'aim congié prendrai.

Renart IV, 387.

7. en espoir de joie avoir me tient cist mals

R. u. P. I, 40, $3^{2}$.

8. je ne me plaing pas des mals, si m'ont greve, por la grant joie ou je be.

Archiv 43, 299.

9. qui me rendroit mon aignel et mon domage, a li me rent.

Salus d'amors bei Jubinal 2, $24 \mathrm{I}$.

10. nule riens a bele dame ne se prent.

La chastelaine de S. Gille (Méon, Fabliaux III) 84.

I1. de vos vient li mals, amie, ke je sent.

Archiv 43, 313.

12. cil deit bien joie mener qui joie atent des max qu'il sent.

Cour de paradis 287.

13. dame, a vous siervir m'otroi tout mon vivant.

Renart le Nouvel 7034.

14. s'aim trop mels un poi de joie a demener que mil mars d'argent avoir et puis plorer.

R. u. P. I, 49, 60 f. 68,9 f.

15. en ma dame ai mis mon cuer et mon penser.

Herrigs Ärchiv 42, 253.

16. je muir, je muir d'amouretes, las aimi! par defaute d'amicte et de merci.

Renart le Nouvel 6766. 7008.

17. je cuidoie avoir ami, ori! ori! mais je ai a tout failli.

Renart le Nouvel 6914. Hier ist mir jedoch die Zusammenfassung ebenso zweifelhaft wie in der folgenden von Diez angeführten Stelle:

18. j'ai au cuer d'amer la raige, aimi! aimi! comment pourrai je tant faire que j'aie ami.

Renart le Nouvel 4608 var. Namentlich bedenklich ist die zweite Zeile.

19. et la mort est au degre qui me deffie.

Histoire littéraire 23,568 .

20. moult hei ma vie, s'a teie tort me fait morir ma douce amie.

Archiv 42, 242. 
21. fine amors loiaus est boene a maintenir.

R. u. P. I, 7 I, 7 .

R. u. P. II, $2 \mathrm{I}, 58$.

22. or ai bone amor novele a mon plaisir.

23. tout li cuer me rit de joie quant la voi.

Dinaux 4, 458, von Jehans Erars.

24. toz li cuers me rist de joie quant dieu voi.

Cour de paradis 487 . Geistliche Parodie des vorigen.

25. ne sui pas les mon ami, ce poise mi,

Renart le Nouvel 6978. 4568 var. Die Versabtheilung ist mir zweifelhaft.

26. entres m'est li maus d'amer ou cuer par l'uel.

Baudouin de Condé 636 .

R. u. P. I, 40, 8.

27. senz amor ne puis durer ne je ne vuell.

28. honis soit maris ki dure plus d'un mois.

R. u. P. II, 27, 30 .

29. batue sui pour amer de mon baron, et si n'en fai nul sanlant se rire non.

Renart le Nouvel 4520.

R. u. P. I, 67, I 8.

30. il n'est deduis ne solas fors ke d'amors.

Jubinal 2, 24I.

31. jai ame et aime encore et ai amours.

32. deus! tant mal mi fait la guaite ki dist 'sus,

or sus, or sus!'

li jors n'est pas venus.

Refrän eines Tanzliedes 'Au nouviau taus' von Moniot de Paris.

Auch sonst noch kommt in der Lyrik diese Versart vor; so in einem Lais von Gautier de Dargies 'J'ai maintes fois chante', wo ein Absatz lautet:

Quant voi sa belle semblance et son vis cler, adont n'a pas esperance de finer,

ains cuide bien ceste enfance ades mener;

mais rois ne porroit en France einsi durer;

also mit Inreim in den Cäsuren und mit Elision über die Cäsur hinüber.

Ebenso ein Absatz in dem 'Lais d'Aelis', mit männlicher und weiblicher Cäsur, theilweisen Inreimen und Elision über die Cäsur.

Fe ne sai s'il est folie ou s'il est sens;

en amer me font gaster amors mon tens.

nuit et jor sospir et plor quant me porpens.

sospirer cele me fait a cui je pens.

diex m'otroit ke ce ne soit sor son deffens.

morir quit se de li n'ai secors par tens.

Mehrere Verse in dem ersten Absatz des 'Lais des amanz', mit weiblichem Cäsurreim und gestatteter Elision.

d'amors est tote la note del sonet,

par amors le chante et note cui bon est... 
et ki plus a cortoisie et plus valor
ke feme ki soit en vie hui cest jor.

Ein anderer Absatz in demselben Lais ist folgendermassen gebildet:

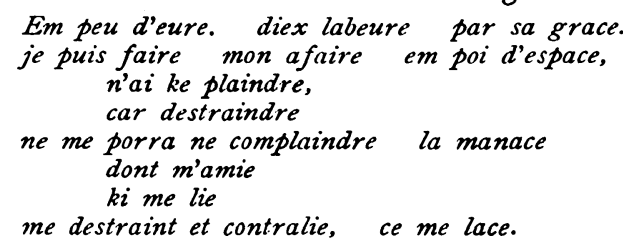

Und so liesse sich noch manches Beispiel aus altfranzösischer Kunstlyrik beibringen; ebenso werden ungedruckte Lieder noch manchen Beleg zum Vorkommen des elfsilbigen Verses in Refräns liefern. Nicht hierher rechne ich solche Beispiele, wie sie Diez S. 125 anführt:

A tous les jors de ma vie la servirai,
et serai en sa baillie tant com vivrai etc.

weil hier bei weiblicher Cäsur die zweite Hälfte doch vier Silben hat, was sonst nur bei Elision vorkommt.

Dass das fragliche Versmass ein altes und zugleich volksthümliches ist, darauf führen folgende Momente:

I. Das Vorkommen bei so alten provenzalischen Dichtern wie Guillem IX. von Poitou, Marcabrun, Raimbaut von Aurenga, von denen die beiden erstgenannten am Anfange der Kunstpoesie stehen und sich noch vielfach an die später aufgegebenen volksmässigen Weisen halten.

2. Das Vorkommen in einem Tanzliede, einer Gattung also, welche ihrer Natur nach an volksmässige Melodien und Rhythmen sich anschliesst.

3. Das Vorkommen in der altfranzösischen Pastourelle, die, wenn auch selbst ein Product der höfischen Kunstpoesie, sich doch in der Form vielfältig an volkstümliche Weisen angelehnt und solche benutzt hat.

4. Die häufige Verwendung in altfranzösischen Refräns, die recht eigentlich aus dem Volksliede stammen, in denen wir Reste altfranzösischer Volkslieder haben.

5. Die Bemerkung von Guillem de Berguedan weist darauf hin, dass am Ende des 1 2. Jahrhunderts diese Versart bereits zu den fremdartigen und veralteten gehörte. Sie war, weil aus der Volkspoesie stammend, der die Kunstpoesie sich mehr und mehr entfremdete, ausser Gebrauch gekommen.

6. Die Umänderungsversuche in der Handschrift $C$, theilweise auch in $R$, zeigen, dass in späterer Zeit das ursprünglich volksmässige Versmass gar nicht mehr verstanden wurde.

7. Die gänzlich andere Auffassung des Verses in den Leys d'amors ist gleichfalls ein Beweis, dass in späterer Zeit der alte Vers in seiner ursprünglichen Gestalt nicht mehr begriffen ward. 
Suchen wir nach dem Ursprunge der Versart, so ist von vornherein ein germanischer gänzlich abzuweisen. Denn zu der Zeit, wo germanische Poesie hätte einen Einfluss üben können, kann von einer solchen Versart im Deutschen überhaupt nicht die Rede sein. Am nächsten liegt es nun, an die lateinische Poesie zu denken. Die römische Poesic zeigt keinen derartig gebildeten Vers, aus welchem der provenzalisch-französische elfsilbige abgeleitet werden könnte. Für den längeren, fünfzehnsilbigen Vers lässt sich allerdings der katalektische trochäische Tetrameter vergleichen, dessen auch schon Diez (S. I 23) gedenkt. Wir wissen, dass der trochäische Tetrameter eine in der römischen Volks- und Soldatenpoesie beliebte Form war. Das würde stimmen; ebenso das Vorkommen in der geistlichen Dichtung des Mittelalters, theils ohne Reim in der Cäsur, theils mit Reim, aus welch letzterer Form eine der beliebtesten Formen der kirchlichen Poesie hervorging. Allein beim lateinischen Tetrameter ist die weibliche Cäsur durchaus weiblich und der weibliche Einschnitt gehört zum Wesen des Verses. Dagegen haben wir gesehen, dass die beiden ältesten Troubadours (Guillem von Poitou und Marcabrun) dem Verse eine männliche Cäsur geben, wobei die zweite Hälfte nur sieben Silben hat, so dass in der Cäsur zwei Hebungen zusammenstossen. Daraus ergibt sich, dass die männliche Cäsur die ursprüngliche ist, wie auch der männliche Reim, und dass nur vertretungsweise auch weibliche stehen darf.

Den kürzeren (elfsilbigen) Vers aus dem lateinischen katalektischen trochäischen Trimeter abzuleiten ist noch viel bedenklicher, weil diese Versart weder bei Griechen noch bei Römern beliebt war, also unmöglich eine volksmässige sein konnte. Den kürzeren Vers als Verlängerung des zehnsilbigen mit Cäsur nach der sechsten Silbe zu nehmen, wie Diez S. 123 geneigt scheint, geht nicht an. Denn im romanischen Verse, der eben nach Silben gezählt wird, ist jede Silbe mehr oder weniger etwas sehr wescntliches. Ein Vers, der vor der Cäsur sieben Silben hat, ist eben ein durchaus anderer als einer mit nur sechs Silben davor. Eher kann man einen siebensilbigen männlich-reimenden mit einem achtsilbigen identificiren, wie diese Identification thatsächlich in der anglonormannischen Poesie sich vollzogen hat ', aus dem Grunde, weil die Zahl der Hebungen dieselbe ist.

In der kirchlichen Poesie des Mittelalters kommt der elfsilbige Vers zuweilen vor, doch nicht häufig. Aus einer Colmarer Handschrift des 12. Jahrh.s theilt Mone (I, 56) einen Hymnus mit, von dem ich nur die beiden letzten Strophen wiederhole:

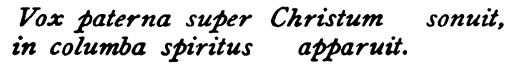

Culpa carens culpas nostras abluit, perit ira, quam vir primus meruit.

' Vgl. auch Jahrb. 12, 2. 4 . 
Die Cäsuren sind auch hier männlich oder weiblich, die Reime nur männlich. Gegen den Rhythmus verstösst V. 2 speciosum prae filiis hominum, wo umzustellen sein wird prae filiis speciosum.

Dieselbe Handschrift enthält noch ein zweites Gedicht, welches noch mehr an die bei den Troubadours übliche Strophenbildung erinnert. Drei Langzeilen beginnen dic Strophe, worauf zwei kürzere Verse folgen. Die erste Strophe lautet (Mone I, 56):

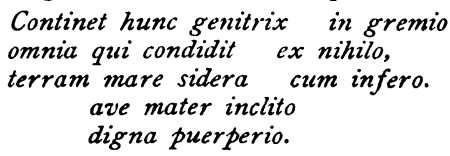

Die Cäsuren sind männlich oder weiblich. Unrichtige Betonung im Reim findet sich in an homo (: animo : pretio) 12 , und in der Cäsur in V. 17. 18. Gleichwohl kann die Identität des Rhythmus nicht zweifelhaft sein.

In einem. Gedichte von Guido de Basoches ( $†$ I 203), das sich in der Handschrift seiner Briefe zu Luxemburg befindet (M. II, 6). Die erste Strophe lautet:

Dei matris cantibus sollempnia
recitat sollempnibus ecclesia:
vota tuis auribus concilia,
te devotis vocibus laudantia,
digna dignis laudibus.
o gloriosa domina,
quorum laudant carmina,
precamur, dele crimina.

Die Cäsuren sind gereimt und durchgängig männlich; die drei letzten Zeilen der Strophe bilden den Refrän. Ganz ähnliche Strophenbildungen haben wir bei den Provenzalen gefunden.

Dann noch in der ersten Strophe eines Gedichtes, das nach Mone von einem französischen Dichter herrührt (M. II, I3 I), in einer Bamberger Hs. des I4. Jahrh.s:

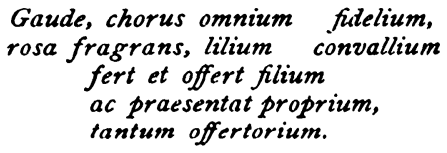

Auch hier sind die Cäsuren der beiden Langzeilen, und zwar mit dem Ende des Verses, gereimt.

Indem ich vorläufig ein fünftes, wichtigstes Beispiel übergehe, wende ich mich zur lateinischen Profanpoesie des Mittelalters. Beachtenswerth ist, dass die von F. du Méril gesammelten 'Poésies populaires latines antérieures au XII ${ }^{\mathrm{e}}$ siècle' (1843) kein einziges Beispiel gewähren. Wohl aber kommen in der Vagantenpoesie des 1 2. und 13. Jahrh. mehrere vor. So Carmina Burana S. 148, wo die ersten beiden Verse elfsilbige Langzeilen mit gereimter Cäsur sind, und kürzere Verse nachfolgen. Der Rhythmus ist in einigen Strophen zerstört, nur die vierte und fünfte sind unverderbt. Die vierte lautet: 


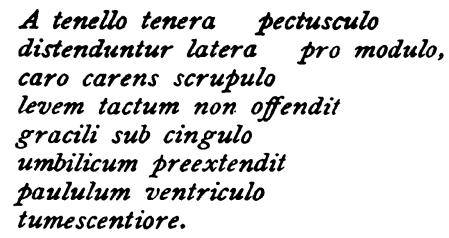

Wenn man hier die VV. 4. 5 und 6. 7 je als eine Langzeile mit Cäsurreim nimmt, so erhält man die Verbindung des elf- und fünfzehnsilbigen Verses, der wir beim Grafen von Poitou begegneten, nur dass hier der längere Vers zweimal wiederkehrt und ausserdem zwei kürzere Zeilen (3. 8) dem Strophenbau angefügt sind.

Ein zweites Beispiel ebenda S. 184 , das folgendermassen $z u$ schreiben ist:

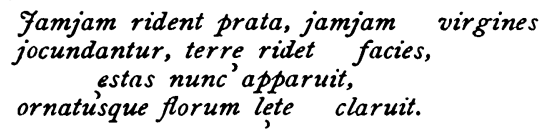

Indess kann man hier zweifeln, wohin die Cäsur fällt. Strophe 2. 3 haben Cäsurreim nach der sechsten Silbe. Allein beweisen kann dies nichts, der innere Reim kann an jede beliebige Stelle gelegt werden, die Cäsur nach der siebenten und achten Silbe ist trotzdem beibebalten. Es sind daher die Verse mit Inreim ebenfalls zu schreiben:

nemus revirescit, frondent frutices,

hiems sera cessit: leti juvenes.

Dass die Cäsur nicht nach 'der sechsten Silbe fällt, ergibt sich aus der Schlusszeile von Str. 2: amor allicit vos jam virginibus. Dass die Zeilen als elfsilbige Langzeilen zu nehmen sind, zeigt endlich die darauffolgende deutsche Nachbildung (S. I 85):

Ih hân gesehen daz mir im herzen | sanfte tuot, des grüenen loubes bin ih worden | wol gemuot, diu heide wunneclîchen stât, mirst liep dazs alsô vil der schoenen | bluomen hâ .

Die Strophe besteht mithin aus drei Langzeilen, in welche eine kürzere Zeile eingeschoben ist.

Auch das Lied S. 201 besteht zum Theil aus dieser Versart, ist aber gleichfalls mehrfach entstellt. Die erste Strophe lautet:

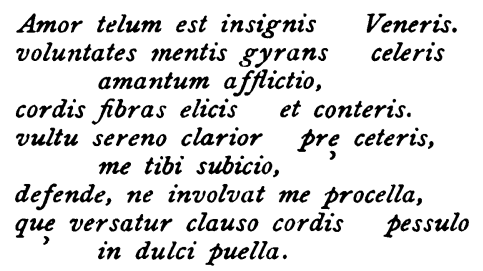

Hier sind V. r. 2. 4. 5. 8 deutlich elfsilbige Verse mit regelrechter theils weiblicher, theils männlicher Cäsur ' ${ }^{\text {; }}$ die drittletzte Zeile ist

1 V. 5 hat eine Silbe zu viel in der vorderen Hälfte. 
ein Endecasillabo, drei kürzere Verse sind ausserdem angereiht. Die deutsche Nachbildung (S. 202) gibt nur die ersten sechs Zeilen wieder.

\section{Ferner S. 2 10:}

Volo virum vivere viriliter.
diligam, si diligar equaliter.
sic amandum censeo, non aliter.
hac in parte fortior, quam fupiter
nescio procari
commercio vulgari;
amaturus forsitan
solo prius amari.

Vier Langzeilen mit durchaus männlicher Cäsur, mit vereinzelten Cäsurrcimer (2, 3. 4. 3, I. 2) bilden den Anfang der Strophe, worauf kürzere Zeilen folgen. Die ganze Strophe ist genau dieselbe, die wir in der Tenzone zwischen Guillelm Raimon und Aimeric von Peguillan (S. 199) fanden; der Unterschied besteht in einer Silbe, die fünfte Zeile hat in dem lateinischen Liede nur sechs statt sieben Silben. Die deutsche Nachbildung (S. 2 I I) hat die Cäsur nicht beibehalten; sie ist zu schreiben

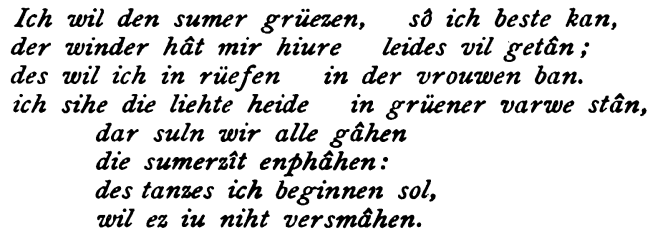

Die Elision über die Cäsur hinüber haben wir in Z. 4 hier ebenso wic im Romanischen. An die Zeile der Nibelungenstrophe darf man bei aller äusserer Aehniichkeit nicht denken; das lateinische Gedicht macht es unmöglich.

S. 224, No. 159 lautet die erste Strophe:

$$
\begin{aligned}
& \text { Vacillantis trutine libramine } \\
& \text { mens suspensa fluctuat et estuat, } \\
& \text { in diversa rapior, } \\
& \text { ratione cum Dione dimicante crucior. }
\end{aligned}
$$

Wir haben hier vollständig die Strophenform des Grafen von Poitou und Marcabruns, nur dass eine kürzere Zeile (V. 3) eingeschoben ist. Die Cäsuren der beiden ersten Zeilen sind mit dem Ende gereimt; die längere Zeile (V. 4) hat Inreim nach der vierten und achten Silbe, genau wie wir es bei Marcabrun (S. 196) fanden.

S. 233, No. 174 beginnen die zweite und vierte Strophe mit zwei elfsilbigen Versen, worauf zwei kürzere folgen:

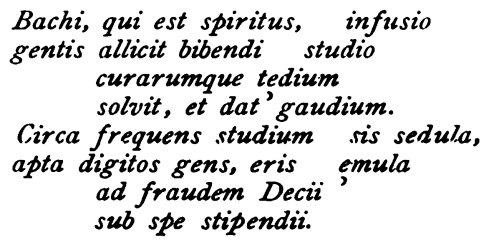

Zeitschr. f. rom. Ph. II. 
Die Strophen sind nicht gleichgebaut, die kürzeren Verse weichen $a b$, und es ist das ganze Gedicht in Leichform.

Seite 227, No. 164 ist der Schluss der Strophe und der Refrain aus elfsilbigen Versen gebildet, während kürzere vorangehen:

Transit' nix et glacies,

spirante favonio,

terre nitet facies

ortu' florum vario, et mihi materies

amor est, quam sentio ad gaudia.

temporis nos ammonet lascivia.

Auch hierzu fanden wir die Analogie in einer altfranzösischen Pastourelle, die gleichfalls mit siebensilbigen Versen begann.

Eine einzelne elfsilbige Zeile in der Strophe S. 203 f., in der Cäsur und Ende reimen:

$$
\begin{aligned}
& \text { inplicat, quam replico et precino. } \\
& \text { provocant me talia ad gaudia. } \\
& \text { per quam mestus vigeo et gaudeo. }
\end{aligned}
$$

Die Schlusszeile jeder Strophe S. 24 I f.:

$$
\begin{aligned}
& \text { cui paupertas semper servit libere. } \\
& \text { cui sors magis aut fortuna faveat. } \\
& \text { per quam nobis cutis erit morbida. }
\end{aligned}
$$

In einem leichartigen Gedichte (S. 132) beginnt der crste Absatz:

$$
\begin{aligned}
& \text { Estatis florigero tempore } \\
& \text { sub umbrosa residens arbore } \\
& \text { avibus canentibus in nemore, } \\
& \text { sibilante serotino frigore. }
\end{aligned}
$$

Hier sind die beiden letzten Verse regelrecht, die Cäsur männlich oder weiblich; die beiden ersten haben mäınliche Cäsur, trotzdem nur drei Silben in der zweiten Hälfte, also dieselbe Erscheinung, die wir bei der längeren Versart auch wahrnahmen, wo ohne Unterschied der Cäsur die zweite Hälfte gleiciımässig sieben Silben hat (S. 196).

Ob man eine Strophenbildung wie folgende (S. 8)

$$
\begin{aligned}
& \text { Vite perdite me legi subdideram, } \\
& \text { minus licite dum fregi quod voveram, } \\
& \text { et ad vite vesperam } \\
& \text { corrigendum legi, } \\
& \text { quicquid ante perperam } \\
& \text { puerilis egi }
\end{aligned}
$$

auch hierher rechnen darf, hängt davon ab, ob nach weiblicher Cäsur des elfsilbigen Verses vier Silben (bei Nichtelision) in der zweiten Hälfte gestattet sind (vgl. S. 202). Dass aber Inreim hier anzunehmen ist, lehrt die zweite Strophe, in welcher die Stellung der inneren Reime wechselt.

Alle bisher angeführten lateinischen Beispiele sind weit jünger als die Lieder des Grafen von Poitou und Marcabruns. Aelter, und zwar um ein Bedeutendes, ist ein kirchliches Gedicht, welches ich seiner Wichtigkeit wegen vollständig mittheile (Mone I, 367).

${ }^{1}$ Hs. Transiit. 
Hymnum luricae. ${ }^{1}$

Subfragare trinitatis unitas, unitatis miserere trinitas, subfragare mihi, quaeso, posito maris magni velut in periculo,

5 Ut non secum trahat me mortalitas hujus anni neque mundi vanitas; et hoc iden peto a sublimibus caelestis militiae virtutibus,

$\mathrm{Ne}$ me linquant lacerandum hostibus,

Io sed defendant jam me armis fortibus,

- et me illi procedant in acie caelestis exercitus militiae,

Cerubim et seraphim cum millibus Gabrihel et Michahel similibus;

15 opto thronos, virtutes, archangelos, principatus, potestates, angelos,

Ut me denso defendentes agmine inimicos valeam prosternere. tum deinde caeteros agonithetas,

20 patriarchas quatuor, quater prophetas, Apostolos, navis Christi proretas, et martyres omnes peto athletas, ut memet per eos salus sepiat atque omne malum a me pereat.

25 Christus mecuin pactum firmum feriat, timor, tremor tetras turbas terreat. inpenetrabili, deus, tutela undique defende me potentia.

Mei gibbae pernas omnes libera,

30 tuta pelta protegente singula, ut non tetri daemones in latera mea librent, uti solent, jacula:

Gigram, cepphale cum iaris et conas patam, liganam et sennas, michinas,

35 chaladum, charassum, madianum, talias, batma, exugiam, binas edumas.

Meo ergo cum capillis vertici galea salutis esto capiti,

' Die Stropheneintheilung rührt von Mone her. 10 me fehlt. 15 virtutes Mone] viventes Hs. 20 quatuor 22 Hs. anathletas. 23 memet] me. 29 gybrae Hs. $32 \mathrm{ut}$; Mone will iacula viersilbig lesen, wie V. Io iam $2 w e i-$ silbig. 34 liganam atque sennas michi: nas. 35 verderbt; um zwei Silben zu lang. 36 atque binas. 37 et vertici. 
fronti, oculis, cerebro triformi,

40 rostro, labro, faciei, tympori;

Mento, barbae, superciliis, auribus, genis, buccis, internaso, naribus, pupillis, rotis, palpebris, tautonibus, gingis, anhelae, maxillis, faucibus;

45 Dentibus, linguae, ori, uvae, gutturi, gurgulioni et sublinguae, cervici, capitali, centro, cartilagini, collo clemens adesto tutamini.

Deinde esto lorica tutissima

50 erga membra, erga mea viscera, ut retrudas a me invisibiles sudum clavos, quos figunt odibiles.

Tege ergo, deus, forti lorica cum scapolis humeros et brachia,

55 tege ulnas cum cubis et manibus pulmos, palmas, digitos cum unguibus.

Tege spinam et costam cum artubus, terga, dorsum nervosque cum ossibus, tege cutem, sanguinem cum renibus,

60 catacrines, nates cum femoribus.

Tege gambas, sura, femoralia, cum genuclis poplites et genua, tege talos cum tibiis et calcibus, crura, pedes plantarum cum basibus.

65 Tege ramos concrescentes decies cum mentagris, unges binos quinquies, tege pectus, jugulam, pectusculum, mamillas, stomachum et umbilicum.

Tege ventrem, lumbos, genitalia,

70 tege alvum et cordis vitalia, tege trifidum jecor et ilia, marsim, reniculos cum obligia.

Tege toliam, thoracem cum pulmone, venas, fibras, fel cum bucliamine;

75 tege carnem, unguinem cum medullis, splenem tortuosis cum intestinis.

Tege vesicam, adipem et pantes conpaginum innumeros ordines; tege pilos atque membra reliqua, 80 quorum forte praeterivi nomina.

39 et cerebro. 40 labio. 44 et faucibus. 46 sublingua Hs. 70 tege fehlt. 71 triphydum Hs. 72 vor cum noch fethrem. 
Tege totum me cum quinque sensibus et cum decem fabrefactis foribus, ut a plantis usque in summum verticem nullo membro foris ego aegrotem,

$85 \mathrm{Nec}$ de meo vitam possint tradere pestis, febris, languor, dolor corpore, donec jam donante deo seneam et peccata mea bonis deleain;

Ut de carne iens imis caream

90 et ad alta evolare valeam, et miserto deo ad aetherea laetus vehar regni refrigeria.

Explicit hymnus, quem Lathacan scotigena fecit.

Die von Mone zu Grunde gelegte Handschrift (in Darmstadt No. 2 ro6) ist aus dem 9., nach Mone aus dem Ende des 8. Jahrhunderts, und bezeugt ausdrücklich in der Unterschrift keltischen Ursprung. Die Behandlung der Cäsur ist wie im romanischen Verse, d. h. männlich nach der siebenten, weiblich nach der achten Silbe, in den weitaus meisten Versen. Doch erlaubt sich der Dichter manche Freiheiten in der Betonung, indem er dreisilbige Worte mit vorletzter Länge auf erster und dritter Silbe betont, prócedánt I I, ebenso virtutés 15; adestó 48; loricá 49; nervosqué 58; plantarúm 64; thoracém 73; zweisilbige auf der letzten: figuint 52; cubís 55; costám 57; cordís 70; jecór 71; mehrsilbige mit kurzer vorletzter auf dieser: cerébro 39; adipem 77 ; innuméros 78. Dieselbe Erscheinung findet sich auch im Reime; vgl. próretás : athletas 2 I f.; tútela (: potentia) 27; conás (: michinas) 33 ; triformi (: tympori) 39 ; loricá (: brachia) 53 ; umbilicuim (: pectusculum) 68; médullis : intéstinis 75 f.; pantés (: ordines) 77 ; ágrotém (: verticem) 84 . Fin paar mal findet sich Synaloephe von unbetontem $i$ im Hiatus; vgl. 41. 46. 73. Elision nur in V. 45 und, wenn meine Frgänzung richtig, in V.83. Der Reim ist durchaus männlich, die Verse paarweise gereimt; nur $19 \mathrm{f}$. begegnet weiblicher Reim (agonithétas: prophétas). Ob man in den Versen, in welchen vor der Cäsur eine Präposition steht, Trennung derselben von ihrem Substantiv durch die Cäsur annehmen soll, ist zweifelhaft. So in maris magni velut in / periculo V.4; vgl. noch 7. 60. 72. 76. 91. Zweifelhaft deswegen, weil entschieden Verse vorkommen, in denen die Cäsur nach der sechsten und, wenn männlich, nach der fünften Silbe fällt. capitali, centro | cartilagini, V. 47 ,

und so noch 51. 61. 69. 92; nach der fünften venas fibras fel | cum bucliamine.

83 summum fehlt. 84 ego fehlt. 87 dante; auch hier müsste man iam zweisilbig lesen. 
Danach wird man auch die Verse, in denen die Präposition auf die siebente Silbe fällt, zu denen mit veränderter Cäsur rechnen dürfen, und abtheilen

$$
\text { et hoc idem peto | a sublimibus etc. }
$$

Ein noch älteres Beispiel dieser Versart gewährt ein Evangeliarium der fürstlich Wallersteinischen Bibliothek $\mathrm{zu}$ Maihingen, welches Wattenbach im Anzeiger für Kunde der deutschen Vorzeit, October I 869, Sp. 289-293 beschrieben hat. Auf der Innenseite des ersten Blattes stehen unter der Aufschrift 'Kanon euangeliorum' lateinische Verse über den Kanon, von welchen Wattenbach den Anfang mitgetheilt hat, und von denen ich eine vollständige Abschrift der oft bewährten Gefälligkeit des Freiherrn von Löffelholtz verdanke. ${ }^{1}$

I. Quam in primo speciosa quadriga homo leo vitulus et aquila

$L X X I \quad \mathrm{LXX}$ unum per capitula de domino conloquntur paria.

II. In secundo subsequente protinus homo leo loquitur et vitulus quibus inest ordinate positus

CVIIII centum in se atque VIIII numerus.

III. Tum deinde tercio in ordine homo et bos loquitur cum volucre 10

$X X I I$ numero quo consistunt antiquae albabeti ebreorum litterae.

IIII. Quanto loco fatentur aequalia una leo homo atque aquila

$X X V I$ uno ore loquentes kapitula verbi summi sena atque vicena.

V. Quinta vice concordant in loquela homo prudens atque mitis hostia

$L X X X I I I$ Jhū xp̄i emicantes agmina Juda sine salvatori (sic) credula

VI. Ecce sexto pari sonant clamore natus adam cum clamoso leone. computata traditis pro munere

$X L V I I I$ Sacerdotum oppidis in honore.

VII. En loquntur septies in septimo

VII homo avis consona de domino

VIII. In octavo nunc leonis catulus dei verba profert atque vitulus

$X I I I$ quorum simul computatur numerus adiecto paulo apostolicus.

' Die cursiv gedruckten Zahlen am Rande sind in der Handschrift roth.

I I eine Silbe fehlt; vielleicht consistebant? oder ist anticuae viersilbig auszusprechen. 30 um eine Silbe zu kurz; sollte der Verf. adiecto viersilbig gesprochen haben? 
VIIII. Nonus ordo in quo duo pariter conloquntur vitulus et volucer

$X X I$ Inspirati sensu spiritaliter proloquntur ternum septipliciter

$\mathrm{X}$. homo nimpe verbum profert proprium 35

LXII Sexaginta et per duo numerum. Rugientem q. leonem audies.

$X V I I I I$ Solum sane decies et novies bovem solum fatentem invenies

$L X X I I$ verba dei bis et septuagies subvolantem ad astra repperies

$X C V I I$ nonagies loqui atque septies.

Auch hicr die ganz gleiche Behandlung des Verses, überwiegend (lic Cäsur nach der siebenten oder achten Silbe, dieselben Freiheiten der Betonung in der Cäsur (cónsistint I I; fátentür I 3; lóquevités I5; cóncordánt I7; fátenlém 39; astrá 41) und im Reime (quádrigá I; vicená 16 ; lóquelí 17 ; clámors : léoné $21 \mathrm{f}$; hónoré 24). Der Reim durchaus männlich, die Bindung überwiegend vierfach, seltener gepaart; die Cäsur zuweilen nach der sechsten Silbe (30. 32. 44), einmal nach der fünften (40); auch hier einmal die Präposition an siebenter Versstelle (3), wo also auch die Cäsur nach der sechsten Silbe anzunehmen sein wird.

Das hier bemerkte Schwanken in der Cäsur macht mich noch mehr geneigt, auch für das Romanische einen Langvers anzunehmen, der nach der fünften (sechsten) Silbe die Cäsur hatte (vgl. S. 202). Nur hat sich, was bei den Kelten innerhalb derselben Versart geschehen durfte, bei den Romanen in zwei getrennte Versarten gesondert; der Vers musste entweder die Cäsur nach der fünften (sechsten) oder nach der siebenten (achten) Silbe haben und innerhalb desselben Gedichtes musste die eine Art beibehalten werden. Genau so finden wir es in der Entwickelung des zehnsilbigen Verses; nur I.yriker gestatten sich die Verlegung der Cäsur von der vierten zuweilen nach der sechsten Silbe, insbesondere die Italiener, die in provenzalischer Sprache dichteten.

Andere alte Beispiele aus dem Irischen sind mir nicht zur Hand; erwähncn will ich indess, dass in einem irischen sehr alten Missale sich eine lateinische Litanei findet, deren einzelne Abschnitte mit den Worten beginnen:

$$
\text { Peccutores te rogamus audi nos, }
$$

worin ich ebenfalls jenen elfsilbigen Vers wiederzufinden glaube.

Von Gedichten in irischer Sprache weiss ich keins und wusste mir auch Windisch keins anzugeben, in welchem dieser Vers begegnet. ${ }^{1}$ Mir fiel vor etwa fünf Jahren eine deutsche Uebersetzung, ich weiss nicht ob irischer oder anderer keltischer Gedichte, in

38 Hs. noies.

${ }^{1}$ In dem von Zeuss, Gramm. celt. ${ }^{2} 968$ angeführten Gedichte sind vielleicht einige Verse dieses Masses, aber jedenfalls nicht durchgeführt. 
die Hände, und darin fand ich den elfsilbigen Vers genau in der Behandlung wicder, wie sie bei Provenzalen und Franzosen vorkommt. Leider ist mir das Blatt, auf welchem ich mir ein betreffendes Excerpt machte, verloren gegangen, und ich habe trotz mehrfachen Nachforschens das Buch nicht wieder auftreiben können.

Dagegen ist die längere Versart, welche wir als Schluss der Strophe auf den elfsilbigen Vers beim Grafen von Poitou und bei Marcabrun folgend fanden (S. I96 f.), in der irischen Poesie eine ganz geläufige Form; eine Langzeile von vierzehn Silben mit einer männlichen Cäsur nach der siebenten Silbe: zwei solcher Iangzeilen bilden eine Strophe. Das nachfolgende Beispiel verdanke ich Windisch.

a n-dorigne do fertaib | ni fail dorurme co cert

amra ro gab prainn Lusdach|trenfer di dé gaib a nert.

Daraus ergibt sich, dass auch im Provenzalischen die männliche Cäsur nach der siebenten Silbe das Ursprüngliche ist, und dass die weibliche nur vertretungsweise dafür eintreten darf. Schon das scheidet den Vers streng vom römischen Tetrameter, dem die weibliche Cäsur nach der achten Silbe unentbehrlich ist.

Auch in lateinischen von Iren verfassten Gedichten finden wir den vierzehnsilbigen Langvers frühe. So in dem Gedichte eines irischen Verbannten an Karl den Grossen, das Zeuss (Grammatica celtica $^{2}$ S. 948) citirt:

Charta Christo comite | per telluris spatium

ad Caesaris regium | nunc perge palatium.

Fer salutem Caesari $\mid$ ac suis agminibus,

gloriosis pueris | sacrisque virginibus. etc.

Eine wie es scheint spätere Entwickelung ist es, dass nicht die Enden der Langzeilen, sondern Cüsur und Ende gereimt werden, wie in folgendem mir ebenfalls von Windisch mitgetheilten Beispiele:

\section{ro bad inmain ld, mad fir ricfed Cuchulaind no thir ram biad arcat ocus or rom biad mor fina do bl.}

Auch hierzu fehlt es nicht an der vollständigen Analogie im Provenzalischen. Wir haben mehrere I.ieder angeführt, in denen die Strophe mit drei oder vier elfsilbigen Versen beginnt, worauf kürzere Verse von sieben Silben, paarweise oder vierfach gereimt, folgen; vgl. S. 197-200. Fasst man je zwei von ihnen als metrische Einheit, als Langzeilen, so haben wir genau den Vers, der beim Grafen von Poitou und Marcabrun ebenfalls die Strophe beschliesst. Auch im Irischen wurden die beiden aus einer vierzehnsilbigen Langzeile aufgelösten siebensilbigen Verse noch als Finheit aufgefasst, wie sich daraus ergibt (worauf mich ebenfalls Windisch aufmerksam macht), dass die Sinnespause nach der zweiten Zeile fällt, also I und 2 , ebenso 3 und 4 dem Sinne nach je eine Einheit bilden.

Stammt, wie nach der vorausgehenden Darlegung wohl nicht bezweifelt werden kann, der elfsilbige und vierzehnsilbige Vers aus 
dem Keltischen, war, wie dic alten lateinischen Beispiele darthun, die elfsilbige Versart ebenfalls bei den Iren in Gebrauch, so sollte: man denken, dass sie in irischer Volkspoesie sich erhalten hïtte. In Thomas Moore's Irish Melodies finde ich nur eine Versart, dic etwa zur Vergleichung herangezogen werden könnte. 'The irish peasant to his mistress' beginnt:

Through grief and through danger thy smile hath cheer'd my way, till hope seem'd to bud from each thorn that round me lay.

Die vordere Vershälfte ist daktylisch, mit einer Vorschlagssilbe; nicht anapästisch, weil sonst zweisilbiger Vorschlag stehen würde. Der Rhythmus ist also

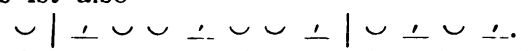

Da auch der romanische Vers nicht selten einen daktylischeri Rhythmus hat, wie der folgende (S. 198)

oder (S. 199)

$$
\text { uina danséta voill fär ljogán rizén }
$$

$$
\text { quél come'nsiirs ab la fin | aj' ácordinisa, }
$$

so ist in der That eine Aehnlichkeit mit dem Verse bei Th. Noore nicht $z u$ verkennen.

Ich zweifle nicht, dass bei näherer Durchforschung der keltischen, insbesondere der irischen Poesie sich der elfsilbige Vers wird nachweisen lassen; aber auch ohne dass er bis jetzt in ihr belegt ist, wird man nach vorliegender Darlegung schon jetzt den keltischen Ursprung nicht nur des vierzehn-, sondern auch des elfsilbigen Verses im Provenzalischen und Französischen behaupten dürfen.

\section{K. BAR'rSCH.}

